\title{
Ripples in Tapped or Blown Powder
}

\author{
Jacques Duran \\ LMDH- UMR 7603 CNRS- Université P. et M. Curie \\ 4 place Jussieu, 75252 Paris Cedex 05 \\ email address : jd@ccr.jussieu.fr
}

May 20, 2018

\begin{abstract}
We observe ripples forming on the surface of a granular powder in a container submitted from below to a series of brief and distinct shocks. After a few taps, the pattern turns out to be stable against any further shock of the same amplitude. We find that the wavelength of the pattern is proportional to the amplitude of the shocks. Starting from considerations involving air flow through the porous granulate and avalanche properties, we build up a semi-quantitative model which satisfactorily fits the set of experimental observations of either tapped or blown powder.
\end{abstract}

In the recent years, there has been a great deal of interest in the response of granular materials to various kinds of external perturbations. Up to now, the vast majority of the experimental, theoretical and simulated works have dealt with model granular solids in the sense[1] that the particles were supposed to be large enough (i.e. typically larger than $100 \mu \mathrm{m}$ ) to avoid significant interaction with the surrounding fluids. In reverse and rather paradoxically, the understanding of the behavior of fine powders has received much less attention although it is universally recognized as the keystone of an increasing number of high-tech industrial processes. In this spirit, a few recent attempts were made towards the analysis of the behavior of fine cohesive (e.g. [2]) or non-cohesive powders (typically in the range from $1 \mu \mathrm{m}$ to less than $30 \mu \mathrm{m}$ ) or of larger particles submitted to excessive windy conditions, involving saltation such as in desert dunes.

Among others, our present knowledge about instability of layers of granular solids under vertical vibrations is currently firmly established. As a matter of fact, the original paper of Melo et al. (4) has stimulated a series of subsequent papers 5] 6] [7] dealing with several facets of the same problem. In short, all these works converged toward a description of the observed dynamic behaviors in terms of Faraday instability in liquids combined with the specific dynamics of the inelastic bouncing ball.

In a less recent past, the vibrational heaping of a sand pile has motivated a debate 91 [10] [11 dealing with the influence of the air interaction in the phenomenology of sand heaping.

The present work (which duplicates into a small scale laboratory experiment, a real industrial device used to empty powder carrying tankers) reports the observation and analysis of a novel form of surface instability of a thin layer (thickness typically $10 \mathrm{~mm}$ ) of a fine powder ( particle size $10 \mu \mathrm{m}$ ) submitted to a series of vertical shocks from below the container. A relatively simple experiment exhibits two essential features which give a trend to a plausible semi-quantitative theoretical explanation of the process. First, it is observed that, after a few taps, the surface displays a regularly corrugated pattern made of a succession of jointed heaps sitting at the natural avalanche angle. The crucial point here is that any further taps do not induce any significant change 
in the pattern which thus can be considered as a steady state with respect to further vertical shocks. Second, and this is a clue to the understanding of the process, the characteristic wavelength of the pattern is found to be directly proportional to the amplitude of the taps.

Our simple analytical model considers the powder-air interaction. It involves two basic features of the fine granular material : Firstly, the Darcy's law for modelling the air flux through the porous cake of granulate. Secondly, the maximum stability angle of a granulate before generating avalanches. In brief, it is shown that a corrugated surface stands as a more stable state than a horizontal flat surface with respect to air blow from below, because it is easier to eject a particle from a flat surface than from an inclined surface sitting at the avalanche angle. Additionally, the apices of the hills created by air blow are seen to be unstable as compared to both sides of the hills. A couple of further experiments provide a clear view of this last feature.

Several basic characteristic features of the surface instability of a tapped powder layer can be readily observed starting from a simple table-top experiment : We use a cylindrical transparent tube made of leucite or glass. The dimensions of the tube are unimportant. In a typical experiment, this tube can be about $20 \mathrm{~cm}$ long and $2.5 \mathrm{~cm}$ in diameter. We half fill the tube with fine powder (e.g. glass beads, diameter $10 \mu \mathrm{m}$ ). We keep the tube horizontal and rigidly fixed at both ends, with the powder initially set flat and horizontal thus giving a granulate thickness of about $12.5 \mathrm{~mm}$ in the center. We take a heavy metallic or plastic rod and knock gently and repeatedly at a very low pace and at a constant intensity onto the center of the tube from below, applying vertically as brief taps as possible, i.e. letting the rod rebound appreciably after each separate shock. After a few taps (about ten to twenty), the surface, initially flat, smooth and horizontal, turns out to exhibit ripples similar to those reported in Fig. 17a and 1 $1 \mathrm{~b}$. Now, tapping more energetically but still keeping the intensity as constant as possible from one tap to the next, induces a pattern where the mean distance between two successive ripples increase significantly. Furthermore and under energetic tapping, a careful observation of the surface shows that, at every tap, a limited number of particles may be ejected upwards starting both from the apices of the hills and from the small plateaux which happen occasionally between imperfectly jointed hills. Using a flat rectangular box as a container instead of a cylindrical tube, and tapping under the center of the box gives rise to surface patterns similar to Fig. 1 c. Note that similar results can be obtained using rather large and hollows i.e. light particles. Excessive wetness prevents the observation of these surface patterns.

Definitely more reliable information has been obtained in the course of our experiments, using a more sophisticated device. We set a CCD (charge coupled device) camera above the tube in order to record and process the successive patterns obtained during the experiments. Secondly, we used a magnetically driven tapping device and a microphone stuck on the tube in order to monitor the amplitude of the taps applied on the sample. Typical experimental results are reported in Fig. 2

Considering the peculiar geometry of the pattern made of jointed heaps sitting approximately at avalanche angle, we note that the characteristic wavelength $\Lambda$ is determined by the height of the pattern. We call $h_{T}$ the altitude (starting from the bottom of the container) of the apices of the corrugated surface, $h_{B}$ the altitude of the valleys of the corrugated surface and $h_{i}$ the altitude of the initially horizontal surface of the granular layer. $\theta$ is the avalanche angle of the powder, which is about $30^{\circ}$ in our glass beads powder.

The wavelength $\Lambda$ is given by

$$
\Lambda=2\left(h_{T}-h_{B}\right) \cot \theta
$$

where $h_{T}+h_{B}=2 h_{i}$ 
a

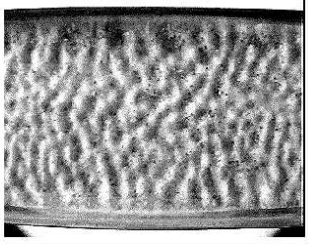

b
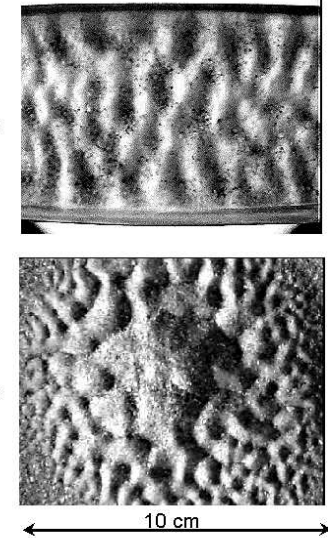

Figure 1: Three bird-eye views of the corrugated surface observed after twenty shocks of constant amplitudes onto the underpart of the containers. Snapshots a and $\mathrm{b}$ corresponds to the cylindrical container and are obtained at two different shock amplitudes, larger in $\mathrm{b}$ than in a. Measurements are performed in the median part of the pattern. Snapshot $\mathrm{c}$ is obtained in a rectangular metallic box (size $20 \mathrm{x} 40 \mathrm{~cm}^{2}$ ) containing a layer of fine sand beach, tapped under the central part. In this latter case, the pattern reproduces the transient deformation of the underlying metallic sheet.

We first start from the first part of an assertion initially put forth by M. Faraday 12 who stated that in the course of vertical vibration of a granular powder "It forms a partial vacuum into which the air round the heap enters with more readiness than the heap itself,..." but instead of following immediately the second part of his statement (stating that it results in sand heaping by carrying sand at the bottom edge with it), we rather consider here that, during the fall following the upward launching of the sand by the taps, the trapped air is forced upwards through the porous medium. Then, supposing for the sake of simplicity that the air flux through the medium obeys a Poiseuille flow, the Darcy's law holds true. It gives the velocity $v$ of a fluid flux emerging from the porous medium whose permeability is $K$ and where the air is pushed upwards by a uniform pressure difference $\Delta P$ acting over a thickness $h$ of the granulate as

$$
v_{h}=K \frac{\Delta P}{h}
$$

In the course of our experiments $\Delta P$ is proportional to the tap amplitude applied on the underpart of the tube $A$ so that the velocity of the air emerging from the surface at altitude $h$ can be written as $v_{h}=\alpha A / h$

where $\alpha$ is the coefficient of proportionality given by Darcy's law which involves the permeability of the granular material.

A single spherical bead (diameter $D$ ) is lifted up by the air flow if the air velocity is larger than a minimum velocity $v_{f}$ ( $f$ standing for free fall velocity) given by $v_{f}=\frac{D^{2}}{18 \eta} \rho g$ where $\eta$ is the viscosity of the gas (air), $\rho$ is the 


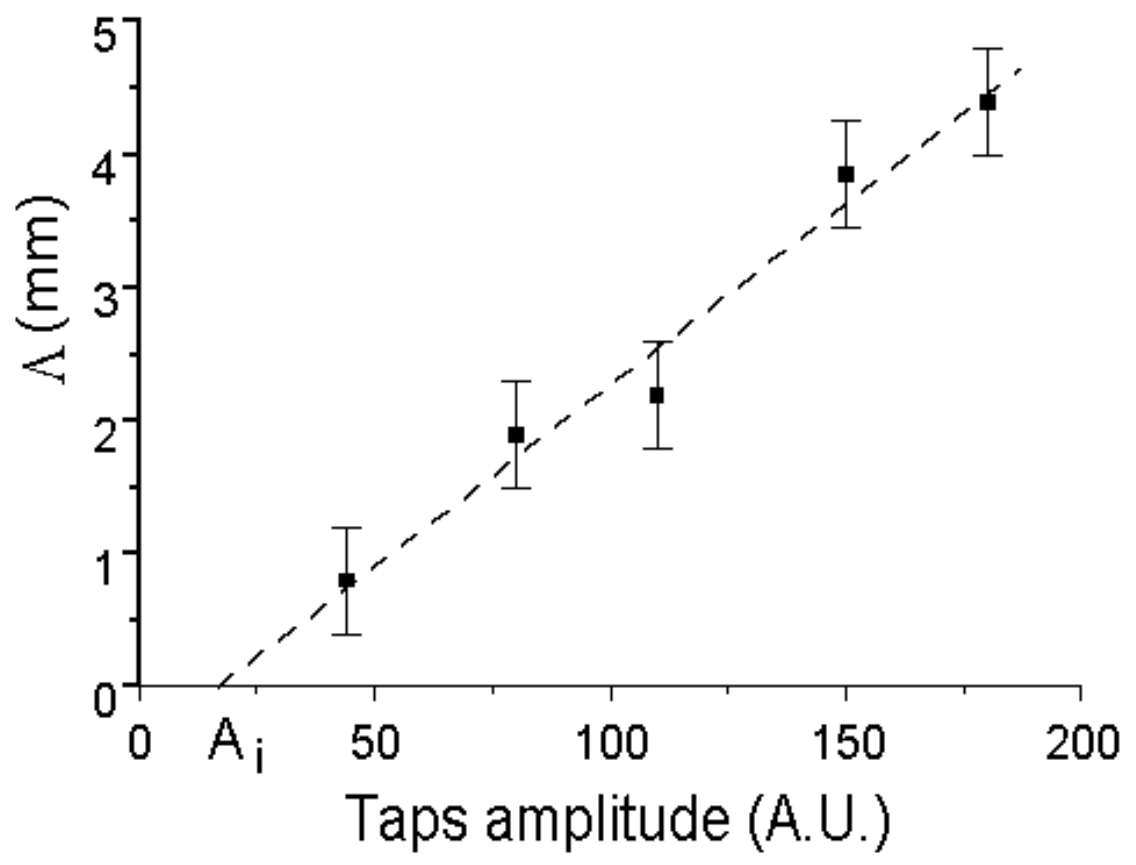

Figure 2: Characteristic wavelength of the pattern in millimeter versus taps amplitude measured as the signal delivered by the microphone in tens of millivolts. The dotted line shows a linear fit of the experimental results.

volumetric density of the particles and $g$ the gravitational acceleration.

Thus a particle deposited on the surface at altitude $h$ can be expelled from an horizontal surface of the layer if the outgoing air velocity is $v_{h} \geq v_{f}$. We find that for glass particles of diameter $=10 \mu \mathrm{m}$, this threshold air velocity is about $1 \mathrm{~cm} / \mathrm{s}$. Thus the threshold quantity $\alpha A_{i}=h_{i} v_{f}$ required for external taps able to blow up particles from the surface of the granulate is about $1 \mathrm{~cm}^{2} / \mathrm{s}$.

First, in view of our experimental observation, we easily realize that the air slowing down process through the granular layer is unable to account alone for the above described observations. If we imagine that the incoming air pulse is unable to eject particles when reaching the apices of the hills, we have $\alpha A_{T}<h_{T} v_{f}$

Calculating the ratio of the required initial velocity to induce hills of height $h_{T}$ to the required initial velocity for the onset of the corrugation, we get $A_{T} / A_{i c}=h_{T} / h_{i}$

Our experiments show that the ratio $h_{T} / h_{i}$ is only marginally larger than 1 while the observed amplitude ratio is about 8 (Fig. 2). We conclude that another process should be taken into account to explain the observed features.

Now we can get an estimated value of the product $\alpha A$ in the course of our experiments. We take profit of our observation that, in the bottom of the valleys when a small flat surface survives or on the top of the hills when the steady state pattern is obtained, a small number of particles are expelled at every tap (See Fig. 3)

In the course of our experiments and near the maximum tap intensity, the upward jump of these particles is on the order of a two tenth of a millimeter. There, the velocity of the emerging particles is given by $\sqrt{2 h_{m} g} \simeq 6 \mathrm{~cm} / \mathrm{s}$ which can be considered as approximately measuring the velocity of the emerging air flux at altitude $h_{m}$ (about $1.2 \mathrm{~cm}$ ). Thus, we find $\alpha A \simeq 7.2 \mathrm{~cm}^{2} / \mathrm{s}$ which in view of Fig. 2 stands as a correct order of magnitude. Note again that due 
to the fact that $h_{T} / h_{B}$ is only slightly larger than one, the outgoing air flux is practically the same at any point on the corrugated surface.

It ensues that on both sides of the hills, the velocity of the air flux propagating upwards is not sufficiently damped when reaching the inclined surface to ensure the stability of the pattern. There, another process must come into play. We put forward the following considerations :

On both sides of the hills, the ascending air flux meets an inclined sheet of particles which is on the verge of avalanching. Under these circumstances, one particle subjected to the vertical incoming air flux bears a fraction of the additional weight of the above lying particles involved in the avalanche layer (see insert in Fig. 3)

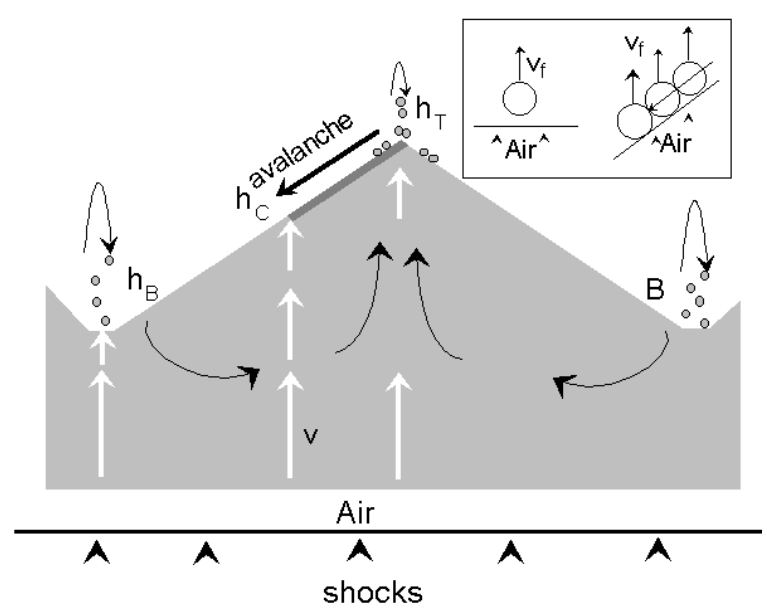

Figure 3: Sketch of the ripples buildup showing the screening effect of the inclined sides of the hills with respect to air blow from below. The white arrows correspond to air trajectory while the black ones show the movement of the particles. Insert : the balance of forces on a horizontal and on an inclined surface.

This additional mass opposes the blowing up of the particles near the surface and therefore stabilizes the inclined lateral surfaces against the incoming air flow. We can build up a simplified equation for this screening effect considering that the mass of the concerned particle is increased by a factor $N p \sin \theta$, $N$ being the number of the above lying particles pertaining to a single sheet of the inclined granulate and $p$ being the unknown number of sheets possibly involved in the avalanche process. Strictly speaking these particles participating to the screening effect need not move, i.e. fall in an avalanche process. They need only to be mobile enough to participate to the mass balance equation involved in the process. Considering that all the particles sitting above the considered particle sitting at altitude $h$ participate to the screening effect, we have $N p \simeq\left(h_{T}-h\right) p / D$. Thus the required air velocity $v_{a h}$ to eject the considered particle sitting at altitude $h$ is given by $v_{a h}=v_{f}\left(h_{T}-h\right) p \sin \theta / D$

As expected, this screening effect determines an altitude $h_{C}$ under which all the particles sitting on the inclined lateral surface cannot be expelled by the air flux. This altitude $h_{C}$ is given by the equation :

$$
\alpha \frac{A-A_{i}}{h v_{f}} \frac{1}{\frac{h_{T}-h_{C}}{D} p \sin \theta} \simeq 1
$$

In other words, the upper part of the hill ( when $h_{C}<h<h_{T}$ ) is unstable 
whereas the lowest one ( when $h_{B}<h<h_{C}$ ) is stable against vertical air blow from below.

Now, in view of several preceding observations of convective processes in vibrational sand heaping (e.g.[11]) experiments and in agrement with $\mathrm{M}$. Faraday's 12 second part of the statement, we note that the steady state of the pattern should result from the balance between the small number of expelled particles near the apices and the number of particles which are reinjected into the bulk of the hills at every taps (see black arrows in Fig. 3). Starting from this observation, we conjecture that this sort of trappingdetrapping process should be independent of the size of the hills. Thus, we write $h_{T}-h_{C}=C\left(h_{T}-h_{B}\right)$ where $C$ is the proportion of the unstable part of the hills. It is an adimensional constant independent of the height of the hills and of the amplitude of the shocks. With this extra assumption, we get the characteristic wavelength $\Lambda$ of the pattern which is proportional to the amplitude of the shocks in agrement with the measurements reported in Fig.2

$$
\Lambda \simeq 2 \frac{\alpha}{C} \frac{A-A_{i}}{h v_{f}} \frac{D}{p \sin \theta} \cot \theta
$$

A numerical estimate using our experimental results is illustrative. We find that $C=25 \%$ of the hills are unstable if only one single layer of powder is involved in the process. If, now, 5 layers of the superficial sheet participate to the process as has been repeatedly observed in avalanche experiments, we see that only $5 \%$ of the upper part of the hills are unstable against the incoming air flux.

The delicate question of the stability of the particles sitting near the apices of the pattern has motivated a further experiment which we performed firstly in order to prove directly the validity of our model based on air-powder interaction and secondly to provide a visual insight into the question of the stability of the apices of the air built pattern.

We use a millipore filter, commonly used in chemistry for filtering, in a reverse manner. A plastic filter (pores $3 \mu \mathrm{m}$ ) is placed at the bottom of a commercial cylindrical glass vessel which allows direct observation or image processing with a CCD camera. In contact with the horizontal filter and above it, we lay a thin layer of powder (about $8 \mathrm{~mm}$ thick). Instead of sucking up through the filter as is usually done, we blow from below, using either brief air pulses or a continuous air flux.

The photographs of the resulting surface corrugation are reported in Fig. 4. The upper snapshot shows up a surface corrugation made up of triangular shaped ripples quite similar to the previously reported in our tapping experiments (Fig. 1). We again observe qualitatively that the stronger the air pulses, the larger the characteristic wavelength of the pattern is.

The lowest snapshot corresponding to a gentle and continuous air flow going through the powder cake is quite informative. Then the surface corrugation exhibits a different aspect because the system has no time to relax between separate successive perturbations as in the preceding experiments. This experiment shows up a myriad of stable small volcanoes organized around small craters (seen as black spots in the snapshot) which spew out powder particles. This can be seen as a consistent support to our preceding picture which involved the relative weakness of the apices of the patterns against air blow from below.

Furthermore, we noted that the building of patterns obtained by tapping the underpart of a granular powder is by no means a reversible effect. For example, applying successively two series of taps at two different and constant amplitudes gives rise to complex patterns which depend on the order of the series. Doing several experiments of this type, we were able to reproduce wrinkled volcanoes patterns or herringbone shaped structures which strikingly remind one of their large scale counterparts in mountainous landscapes. We postpone the description of these findings to a forthcoming paper. 


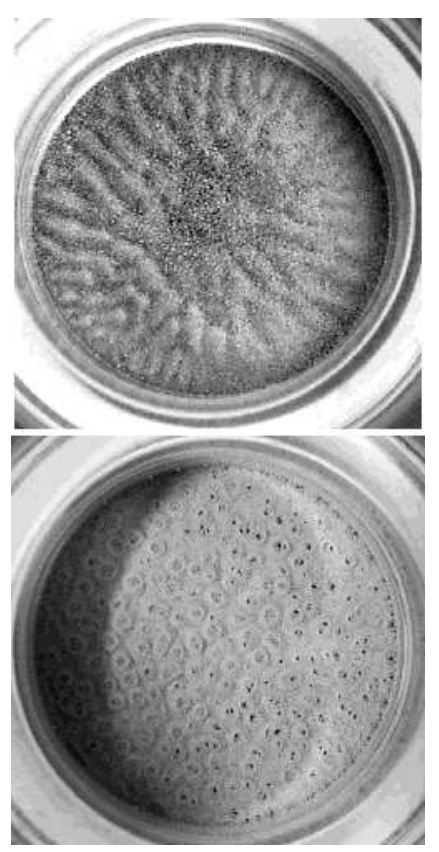

Figure 4: The upper photograph show the ripples obtained by using fast transient air pulses. The lowest photograph obtained with a continuous air flow exhibits small craters (black points) sitting at the center of the myriads of small fixed volcanoes.

I acknowledge fruitful discussions with the granular group in Jussieu, with P.-G. de Gennes and with E. Raphael in College de France.

\section{References}

[1] R. L. Brown and J. C. Richards, Principles of Powder Mechanics, Pergamon Press, Oxford, 1970.

[2] A. Castellanos, J. M. Valverde, A. T. Perez, A. Ramos, and P. K. Watson, Phys. Rev. Lett. 82, 1156 (1999).

[3] T. Raafat, J. P. Hulin, H. J. Herrmann, Phys. Rev. E 53, 4345, (1996)

[4] F. Melo, P. B. Umbanhowar, and H. L. Swinney, Phys. Rev. Lett. 72, 172 (1994).

[5] P. B. Umbanhowar, F. Melo, and H. L. Swinney, Nature 382, 793 (1996).

[6] F. Melo, P. B. Umbanhowar, and H. L. Swinney, Phys. Rev. Lett. 75, 3838 (1995).

[7] E. Clément, L. Vanel, J. Rajchenbach, and J. Duran, Phys Rev. E 53, 2972 (1996).

[8] L. S. Tsimring and I. S. Aronson, Phys. Rev. Lett. 79, 213 (1997).

[9] S. Fauve, S. Douady, and C. Laroche, J. Phys. C3 50, 187 (1989).

[10] P. Evesque and J. Rajchenbach, Phys. Rev. Lett. 62, 44 (1989).

[11] E. Clément, J. Duran, and J. Rajchenbach, Phys. Rev. Lett. 69, 1189 (1992).

[12] M. Faraday, Philos. Trans. R. Soc. London 52, 299 (1831). 